\title{
Building a tool to overcome barriers in research-implementation spaces: the Conservation Evidence database
}

William J. Sutherland ${ }^{1,2^{*}}$, Nigel G. Taylor ${ }^{3}$, Douglas MacFarlane ${ }^{4}$, Tatsuya Amano ${ }^{5}$, Alec P. Christie $^{1}$, Lynn V. Dicks ${ }^{6}$, Anaëlle J. Lemasson, Nick A. Littlewood ${ }^{1}$, Philip A. Martin ${ }^{1,2}$, Nancy Ockendon $^{1}$, Silviu O. Petrovan ${ }^{1}$, Rebecca J. Robertson ${ }^{8}$, Ricardo Rocha ${ }^{1}$, Gorm E. Shackelford ${ }^{1,2}$, Rebecca K. Smith ${ }^{1}$, Elizabeth H. M. Tyler ${ }^{1}$, Claire F.R. Wordley ${ }^{1}$

1 Conservation Science Group, Department of Zoology, University of Cambridge, The David Attenborough Building, Pembroke Street, Cambridge CB2 3QZ, UK

2 BioRISC, St. Catharine's College, Cambridge CB2 1RL, UK

3 Tour du Valat, Research Institute for the Conservation of Mediterranean Wetlands, Arles, 13200, France

4 School of Psychology, University of Western Australia

5 School of Biological Sciences, Goddard Building (8), The University of Queensland, Brisbane, 4072 Queensland, Australia

6 School of Biological Sciences, University of East Anglia, Norwich NR4 7TJ, UK

7 Joint Nature Conservation Committee, JNCC, Peterborough, PE1 1JY, UK

8 School of Biology. University of Leeds, Leeds, LS2 9JT, UK

*Corresponding author E-mail: w.sutherland@zoo.cam.ac.uk

Type of paper: Full length articles (Research papers)

\section{Acknowledgements}


The Conservation Evidence project is or has been supported by AG Leventis Foundation, Arcadia, British Ecological Society, Defra, Economic and Social Research Council, Joint Nature Conservation Committee, MAVA, Natural England, Natural Environment Research Council, The Nature Conservancy, South West Water, Synchronicity Earth and Waitrose. . Dozens of volunteers and staff have contributed to building the Conservation Evidence database. 


\section{Abstract}

2 Conservation practitioners, policy-makers and researchers work within shared spaces with

3 many shared goals. Improving the flow of information between conservation researchers,

4 practitioners and policy-makers could lead to dramatic gains in the effectiveness of

5 conservation practice. However, several barriers can hinder this transfer including lack of time,

6 inaccessibility of evidence, the real or perceived irrelevance of scientific research to practical

7 questions, and the politically motivated spread of disinformation. Conservation Evidence works

8 to overcome these barriers by providing a freely-available database of summarized scientific

9 evidence for the effects of conservation interventions on biodiversity. The methods used to

10 build this database - a combination of discipline-wide literature searching and subject-wide

11 evidence synthesis - have been developed over the last 15 years to address the challenges of

12 synthesizing large volumes of evidence of varying quality and measured outcomes. Here, we

13 describe the methods to enhance understanding of the database and how it should be used.

14 We discuss how the database can help to expand multi-directional information transfers

15 between research, practice and policy, which should improve the implementation of evidence-

16 based conservation and, ultimately, achieve better outcomes for biodiversity.

17 Keywords: evidence-based conservation, evidence-based policy, evidence-based practice,

18 Delphi technique, subject-wide evidence synthesis, research-implementation space

19 Word count: 7790 


\section{Introduction}

Despite efforts to conserve it, biodiversity is being lost at an alarming and increasing

23 rate (Dirzo et al., 2014; Ripple et al., 2017). Research on the effectiveness of conservation

24 interventions is critical to ensure conservation efforts are beneficial, efficient, and not creating

25 additional harms (Cardinale et al., 2012). The number of publications evaluating the impact of

26 conservation-relevant interventions is growing annually, but the lessons learned are often not

27 employed in management decisions or policy (Sutherland et al., 2004; Young and Van Aarde, 28 2011).

This problem has been widely conceptualized as a "research-implementation gap"

30 (Anon, 2007; Knight et al., 2008; Westgate et al., 2018, see Glossary in Supplementary

31 Material). More recently, it has been reconceptualized as an issue within a series of "research-

32 implementation spaces": arenas in which various stakeholders and interest groups interact,

33 collaborate and learn together (Toomey et al., 2017). This concept explicitly recognizes the

34 existing connections between research and practice rather than implying there are voids

35 between research and practice that need to be filled, as well as the broader context in which

36 scientific knowledge is produced and utilized.

Within research-practice and research-policy spaces, several clearly defined barriers

38 limit collaboration and coproduction of knowledge (Roux et al., 2006; van Kerkhoff and Lebel,

39 2015; Table 1). These include communication barriers (e.g. length, linguistic and statistical

40 complexity of scientific articles), financial barriers (e.g. studies hidden behind paywalls),

41 relevance barriers (research often lacks direct relevance to practitioners or policy-makers), 
42 synthesis barriers (an overwhelming volume of unsynthesized scientific literature) and socio-

43 political barriers (e.g. motivated skepticism of information that challenges existing worldviews).

Evidence synthesis is fundamental to overcoming some of these barriers, increasing the

45 flow of ideas within research-implementation spaces, and ultimately helping researchers,

46 practitioners and policy-makers navigate towards the common goal of conserving biodiversity.

47 Evidence synthesis methods aim to locate, collate, and synthesize relevant information, usually

48 from published literature. They range from unsystematic, ad hoc literature reviews, to

49 comprehensive systematic reviews, and even reviews of reviews (Collins et al., 2015). However,

50 these existing approaches have shortfalls. Traditional literature reviews can be subjective, liable

51 to bias and methodologically opaque (Collins et al., 2015; Haddaway et al., 2015). Systematic

52 reviews are designed to reduce those issues, but can be expensive and time-consuming (Borah

53 et al, 2017; Haddaway and Westgate, 2019). Therefore, they are not always possible in

54 conservation, where resources are limited (Soulé, 1985; Gerber, 2016). The intended audience

55 of reviews and systematic reviews sometimes face communication barriers (e.g. Cochrane

56 Clinical Answers are needed as a "readable, digestible" entry point to medical Cochrane

57 Reviews; Cochrane Library, 2019) and financial barriers (e.g. paywalls, although Environmental

58 Evidence provides open access systematic reviews).

To address these issues, we have developed a method to rapidly synthesize evidence

60 across entire subject areas (comprising tens or hundreds of related review questions), whilst

61 being transparent, objective and minimizing bias. Target end users (i.e. researchers,

62 practitioners and policy-makers) are actively involved in the synthesis process. Uniquely, our 
63 subject-wide evidence syntheses (Sutherland and Wordley, 2018) are part of a broader

64 discipline-wide project, pooling resources to increase speed and cost-effectiveness. The

65 ultimate output of this process is the freely accessible, plain-English Conservation Evidence

66 database, which contains evidence for the effects of conservation interventions. The database

67 is complemented by other tools in the Conservation Evidence toolbox (e.g. the journal

68 Conservation Evidence and Evidence Champions). Together, these tools are designed to

69 overcome or lower barriers within research-implementation spaces, increasing the use of

70 evidence in practical conservation and policy-making, and enabling practice and policy to

71 influence research. Ultimately, we hope this will lead to more targeted conservation research

72 and more effective conservation action.

73 In this paper, we focus on the Conservation Evidence database, describing the methods

74 used to create it and how it helps to overcome barriers between conservation researchers,

75 practitioners and policy-makers. Although aspects of the methods have been described

76 previously (e.g. Dicks et al., 2016; Sutherland and Wordley, 2018), this paper provides the only

77 complete and detailed overview of the methods currently used by Conservation Evidence.

78 Through increasing methodological transparency and communicating what the database is (and

79 is not) designed to do, we hope this paper will encourage effective and appropriate use of this

80 tool. We also discuss the database in a broader context, acknowledging that published evidence

81 is just one of a multitude of factors within research-implementation spaces that affect

82 conservation decision making. 


\section{Building the Conservation Evidence Database} within a 1-3 year project and (4) aims and budgets of funders. For example, bat conservation is

\subsection{An overview of the Conservation Evidence database}

The Conservation Evidence database gathers, organizes, and summarizes studies that quantify the effects of conservation interventions (i.e. actions that have been or could be used to conserve biodiversity) on any aspect of biodiversity (e.g. abundance of a focal species, survival rates of translocated individuals, use of nest boxes, extent of habitat) or human behavior related to biodiversity conservation (e.g. levels of hunting, or sales of products detrimental to biodiversity). Ultimately, the database will present the evidence for interventions across the entire discipline of biodiversity conservation. Four key types of information fall largely outside the scope of the database: qualitative data, unpublished practitioner experience, traditional or indigenous knowledge, and detailed information on social or ethical issues (see Section 3.4). At present our focus is restricted to quantitative data which provide objective information on the size and direction of effects.

The database is split into subject areas, usually along taxonomic lines (e.g. bats, amphibians) with some taxa split by habitat (e.g. forest vegetation, shrubland vegetation).

Subjects are distinct areas of research and practice, which we delimit according to (1) what we, and our advisory boards (Section 2.3.2), think would produce a useful synthesis for practitioners; (2) shared conservation challenges and relevance of interventions across the subject; (3) the abundance and distribution of literature, with a subject needing to be covered synthesized separately from conservation of other terrestrial mammals because 
105 chiropterologists form a distinct research and practice community, and because initial funding

106 was limited. At the user interface, subject areas provide a rapid overview of the scope of the

107 database, and coarse filters to focus on the most relevant information.

In each subject area, the database provides a comprehensive list of interventions. For

109 each intervention, the database provides: background information such as the logic behind the

110 intervention and how it might be carried out; standardized paragraphs summarizing individual

111 scientific studies that have quantified the effects of that intervention; key messages that

112 provide a narrative index to the combined evidence from all of those studies; and an overall

113 effectiveness category based on an assessment of the evidence (effectiveness, certainty, and

114 harm) by a panel of experts.

115 In the following sections, we outline the two levels of work involved in building the

116 database and the steps therein (Figure 1). At a high level, discipline-wide literature searches

117 identify publications that fall within the scope of the Conservation Evidence project. At a lower

118 level, subject-wide evidence syntheses collate and summarize studies across subject areas.

\subsection{Discipline-wide literature searches}

\subsubsection{Systematic manual searches}

122 The main method used by Conservation Evidence to find relevant literature is discipline-wide,

123 systematic, manual literature searching. This means identifying literature sources (e.g.

124 academic journals, report series, organizational websites) that are likely to contain relevant

125 information, then manually scanning the title and abstract (or summary) of every document in 
126 those sources. All documents meeting the general inclusion criteria (Section 2.1) are added to a 127 discipline-wide repository, and tagged or filed by subject areas. Sources with a broad, discipline-wide scope (e.g. Journal of Applied Ecology, Biological 129 Conservation) are searched annually to keep the literature repository up to date. Specialist 130 subject sources (e.g. Journal of Mammalogy, British Trust for Ornithology reports) are searched 131 when the synthesis project for that subject is carried out (Section 2.3). Typically, sources are 132 searched from their first publication date until a specified recent date. The list of sources and 133 years screened is published alongside evidence synthesis products. The key advantages of this method are that it does not depend on search term choice 135 and can identify novel interventions not suggested a priori by the authors or advisory board for 136 a synthesis (Sutherland and Wordley, 2018). It is also highly repeatable and transparent, 137 notwithstanding some inevitable variation in the interpretation of inclusion criteria. We use 138 Kappa tests (Cohen, 1960) to identify, and then correct, inconsistency between searchers.

139 Because all relevant publications are added to a discipline-wide repository, each journal issue or 140 block of reports only needs screening once. Each new synthesis or synthesis update can draw 141 from (and contribute to) an existing repository rather than starting afresh (Figure 1), 142 substantially increasing cost-effectiveness. The disadvantages of the systematic manual search approach are that it requires a high 144 initial outlay of time and money, cannot easily incorporate some sources that contain a large 145 number of publications (e.g. mega-journals such as PLOS ONE), and cannot cover sources with a 146 likely low yield of relevant publications. Thus, search terms are used instead of, or to 
147 complement, systematic manual searching in some specific cases (see Section 2.3.4). In the

148 future, automated processes based on machine learning could reduce the cost of systematic

149 source-by-source literature screening, whilst increasing coverage (Westgate et al., 2018).

$150 \quad$ 2.2.2. Non-English and grey literature

152 A large proportion of the global scientific literature in conservation is not published in English

153 (Amano et al., 2016). Conservation Evidence is creating a list of priority conservation journals in

15420 different languages. Of these, 159 journals have been searched by fluent speakers of each

155 language, with more searches underway. Results are being added to the discipline-wide

156 literature repository (Figure 1), with titles and abstracts translated into English. Papers

157 retrieved during these searches are being incorporated into the Conservation Evidence

158 database as staff language skills permit. The aim is to reduce bias in the database towards

159 evidence from English-speaking countries.

"Grey literature" refers to documents not controlled by commercial publishers, such as

161 governmental and non-governmental reports, newsletters, conference proceedings, and theses

162 (Farace and Schöpfel, 2010). Including grey literature in evidence syntheses may help to

163 counteract the problem of publication bias, where studies reporting negative or non-significant

164 findings are less likely to be written up and published in journals (McAuley et al., 2000; Dwan et

165 al., 2013). Conservation Evidence is making a concerted effort to systematically search more

166 grey literature sources (e.g. 687 reports from the British Trust for Ornithology and 945 from

167 Scottish Natural Heritage were searched by 2017) and include relevant publications in the 168 database. 


\subsection{Subject-wide evidence syntheses}

170

171

172 The detailed process of synthesizing evidence for the Conservation Evidence database is broken

173 down into subject-focused work packages, or subject-wide evidence syntheses (Figure 1). The

174 precise subject and scope of each synthesis is decided at an early stage in consultation with the

175 advisory board (see Section 2.3.2). It is essential to define what each synthesis will include and

176 exclude (Pullin and Stewart, 2006). species management or sustainable agriculture. The geographic scope is usually global.

180 Conservation Evidence syntheses are focused on the effects of conservation interventions, so

181 the question structure for review typically follows a PICO format (population, intervention, 182 comparator, outcome). There is a separate review of the evidence for each intervention.

183 Outcome measures are usually direct measures of effects on biodiversity, but may include less

184 direct or intermediate outcomes (see Section 2.1). A synthesis-specific list of focal metrics may 185 be constructed (e.g. abundance of certain indicator plant taxa) to guide consistent reporting of 186 results from summarized studies. Synthesis-specific inclusion/exclusion criteria may also be 187 defined. For example, laboratory and greenhouse studies are excluded for most interventions 188 within vegetation-focused syntheses. 


\subsubsection{Forming an advisory board}

The advisory board for each synthesis is a panel of subject experts who can help to refine its scope (Section 2.3.1) as well as its structure and language (Section 2.3.7), identify

192 interventions (Section 2.3.3) and identify key sources of evidence to search (Section 2.2.1).

193 Advisors contribute to planning each synthesis as well as reviewing a near-final version. Since

194 2018, we have formalized the input of the advisory board to the planning stage by asking them

195 to review a synthesis protocol. These protocols are registered on the Open Science Framework

196 (https://osf.io/mz5rx/) and published ahead of each synthesis on the Conservation Evidence

197 website. We have always used and reported standard methodologies that allow for robust

198 evidence synthesis, but we now appreciate the added value of publishing protocols in advance

199 (Haddaway and Macura, 2018).

Advisory boards are selected to provide expertise in diverse topics within the subject,

201 represent the geographic range covered by the evidence synthesis, and to include a mix of

202 academics, practitioners and policy-makers. Thus, anticipated users of the database contribute

203 to its development, helping to ensure applicability to practice and to increase the likelihood of

204 uptake. So far, advisory boards for Conservation Evidence syntheses have comprised a mean of

$20511.6( \pm 6.9$ SD, $n=15)$ subject experts. Of the 157 individual experts from a total of 28 countries

206 across six continents, approximately 53\% have been from institutes operating more within

207 research domains, and $47 \%$ from organizations oriented more towards policy and practice

208 domains.

\section{2.3.3. Intervention scanning}


211 conservation interventions for the subject of the synthesis is created. The aim is to produce a

212 comprehensive list of all interventions that have been tried or suggested for the subject of the

213 synthesis and that could realistically be implemented. The intervention list can be modified, and

214 added to, as the synthesis process proceeds. Including all possible interventions and then

215 populating these with evidence forms the basis for identifying and mapping evidence gaps (see

216 Section 2.3.6).

Interventions are grouped in a consistent manner across syntheses: primarily according

218 to the IUCN threat category that they address (Salafsky et al., 2008) and, for interventions that

219 tackle multiple threats, secondary categories based on IUCN action types. The naming and

220 division of interventions are guided by both the existing literature and the advisory board.

221 Where possible, interventions are described at a fine scale (for example "Set longlines at the

222 side of the boat to reduce seabird bycatch" is a separate intervention from "Set lines

223 underwater to reduce seabird bycatch"; Williams et al., 2013), so that they can be combined in

224 multiple ways by the user to address larger closed questions (e.g. are longlines at the side of

225 the boat more effective than setting lines underwater to reduce seabird bycatch?), or open

226 questions (what is the state of knowledge on seabird bycatch reduction methods?).

As the evidence synthesis is constructed, background information is added to each

228 intervention. This briefly explains the logic behind the intervention, key issues regarding

229 practical implementation, any unavoidable technical terms used, and potential harms to society

230 or the wider environment. This background information is not, for pragmatic reasons, based on 
231 systematic literature searches and is therefore not taken into account during expert assessment

232 (Section 2.3.8).

\subsubsection{Collating subject-relevant literature}

The synthesis authors collate a repository of literature that is relevant to their synthesis,

235 based on title and abstract/summary screening. The authors draw documents from the

236 discipline-wide repository - which contains the results from screening sources with a discipline-

237 wide focus and sources with a focus on other previously-synthesized subjects (Section

238 2.2.1) - but also search sources most relevant to their synthesis subject (e.g. herpetological

239 journals for an amphibian-focused synthesis). Relevant sources are identified in collaboration

240 with the advisory board. The documents extracted from these synthesis-specific searches in

241 turn feed back into the discipline-wide repository. For example, searches of herpetological

242 journals for the amphibian-focused synthesis will also return papers relevant to a synthesis for

243 reptiles.

244 Conservation Evidence syntheses on a new subject area unlikely to retrieve many

245 publications from the existing discipline-wide repository, or on a very specific subject (e.g. the

246 control of a particular group of invasive species), may use search terms to query databases of

247 scientific literature. In such cases, employing search terms can be a useful complement to, or

248 replacement for, journal searching. If this approach is taken, records are kept and presented to

249 show the databases searched, the terms used and the dates searches were carried out. 
Each publication retrieved through literature searches is screened at full-text by the

253 synthesis authors. If the publication contains at least one study (i.e. conceptually distinct

254 experiment or test of an intervention) that meets the general inclusion criteria as well as any

255 specific criteria defined for that synthesis, then each study is summarized in a standardized

256 paragraph. Reviews and meta-analyses are summarized as evidence if they provide new or

257 collective data relevant to the synthesis.

Summary paragraphs consistently present the same key information from each study in

259 the same order (see Figure A1. in Appendix). This includes: study design; years of study; habitat;

260 location; conservation intervention; target species or habitat; whether there was a statistically

261 significant effect of the intervention and the direction of any effect; quantitative data on the

262 outcome of the intervention; and a brief overview of the methods and monitoring approach.

263 Summary paragraphs are concise - typically around 150-200 words - and written in plain

264 English, avoiding technical terms wherever possible. Although short, the aim is for summary

265 paragraphs to include sufficient detail of the study context and methods to allow users to begin

266 to assess its importance and relevance to their own system (e.g. location, length of monitoring,

267 exactly how the intervention was done) and interpret simple context-dependencies in results.

268 Conservation Evidence does not follow a formal process for critically appraising studies:

269 generally, all studies that meet inclusion criteria are summarized. However, the design and size

270 of each study are reported to help the user - and expert assessors (Section 2.3.8) - judge its

271 importance and reliability (internal validity). As a simple example, the reader might give more

272 weight to results from reviews, and particularly systematic reviews, than to results from 
273 individual case studies. Major concerns (from the original authors or synthesis authors) over the

274 study design are explicitly highlighted in summary paragraphs. Exceptionally, studies may not

275 be summarized if they clearly involve invalid comparisons, or are missing key information that

276 severely inhibits comprehension. These issues are noted in the subject-wide literature

277 repository (Figure 1).

\subsubsection{Key messages: an overview of the summaries}

Summary paragraphs describing studies that test the same intervention are grouped

281 together. "Key messages" provide a brief overview of the studies testing each intervention:

282 usually some indication of the number of studies, their geographical distribution, and their

283 reported effects on key outcome metrics. Key messages are intended to provide an index to the

284 evidence, easing the user into summary paragraphs and helping them identify the most

285 relevant studies to their situation, and to facilitate comparisons of studies.

287 interventions, targets, outcomes, habitats and geographic locations - and thus help identify

288 where further research is needed. For example, no studies were found testing the intervention

289 "Leave unharvested cereal headlands within arable fields" for bird populations (Williams et al.,

290 2013). Furthermore, whilst four studies tested the intervention "Leave standing

291 deadwood/snags in forests" for amphibian populations, they were all carried out in the USA

292 (three in Virginia) and all but one focused on salamanders (Smith and Sutherland, 2014). The

293 key messages across all interventions in a synthesis map the distribution of evidence across the 
294 subject area. Ultimately, key messages across the entire Conservation Evidence database will 295 provide a "mega-map" of evidence for the whole conservation discipline.

We realize our key messages may be interpreted as an invitation to vote count (i.e. draw

297 conclusions based on the number of studies showing positive vs negative results), which is

298 usually a misleading method of synthesis (Stewart and Ward 2019). This is not the intended

299 use. Key messages include information about study designs to suggest that the value of

300 evidence varies between studies. Online, they link directly to the summary paragraphs that

301 contain data to indicate the magnitude of any effects. We have added an explicit warning about

302 vote counting to key messages on the Conservation Evidence website, and are considering

303 alternative ways to provide an overview of the evidence base.

\subsubsection{External review of synthesis}

Once the evidence has been summarized, the draft synthesis is reviewed by the advisory

307 board. They identify problems with language and structure, and suggest further relevant

308 publications not retrieved through literature searches (Sections 2.2.1 and 2.3.4). The synthesis

309 authors then include relevant studies and rectify any problems.

\section{$310 \quad$ 2.3.8. Expert assessment}

Expert assessment is an important final step in synthesizing and presenting the evidence

312 (Figure 1). The aim is to consider studies holistically and generate a generalized, overall

313 effectiveness category for each intervention. This provides users with a supplementary

314 decision-support tool, alongside the key messages and individual study summaries. 
For a Conservation Evidence synthesis, the evidence for each intervention is assessed

316 using a modified Delphi technique (Mukherjee et al., 2015). This involves a panel of

317 experts-academics, practitioners and policy-makers from across the geographic range of the

318 synthesis - carrying out several rounds of scoring for each intervention. The experts score the

319 effectiveness, certainty and harm of each intervention, based on the evidence presented in the

320 synthesis. Anonymized scores and comments are shared within the expert panel between

321 rounds of scoring, to be used as a basis for refining scores. After 2-3 rounds, final median

322 scores are used to assign an overall effectiveness category for each intervention (Sutherland et

323 al., 2018; Table A1 in Appendix).

Effectiveness is scored by considering whether the intervention produces a desirable outcome in the summarized studies, and the magnitude of that outcome $(0 \%=$ not effective,

$326100 \%=$ highly effective). Certainty is a measure of how confident assessors are that the

327 effectiveness score applies across all appropriate contexts. The certainty score incorporates (1)

328 the strength or reliability of the evidence as a whole, based on the number of studies and their

329 quality (internal validity e.g. study design, replication, bias); and (2) how generalizable the

330 results of these studies are, which will depend on the taxonomic/habitat/geographical coverage

331 of studies (external validity). Certainty is scored from $0 \%$ (no evidence) to $100 \%$ (lots of high

332 quality evidence, high generalizability). An intervention could be scored as having high

333 effectiveness if it is supported by many studies showing strong desirable outcomes, but low

334 certainty if those studies use low quality study designs or only consider a specific local context.

335 Harm is scored by rating the magnitude of undesirable effects on the subject of the synthesis

336 from undertaking the intervention $(0 \%=$ no undesirable effects, $100 \%=$ major undesirable 
337 effects). The harm score is important to distinguish interventions that lack desirable effects

338 from those that have undesirable effects: such interventions could receive identical

339 effectiveness (and certainty) scores.

340 Currently, the Conservation Evidence database presents the overall effectiveness

341 categories and the percentage scores for their three components (effectiveness, certainty, and

342 harms). Whilst the percentage scores are useful for giving assessors flexibility and to generate

343 medians across all assessors, we realize they could give a false sense of precision to database

344 users. Thus, in the future, we may move towards categorical groupings to present scores for the

345 three components.

346 Combining evidence from disparate locations, of varying rigor, and reporting different

347 output metrics, is a challenge. Conservation Evidence uses expert assessment rather than meta-

348 analyses to synthesize studies testing the same intervention, but reporting very different

349 metrics, into an overall effectiveness category. For example, expert assessment can combine

350 studies reporting the appearance of Sphagnum moss species on bogs following rewetting (a

351 desirable change) and studies reporting a decrease in tree cover on bogs following rewetting

352 (also a desirable change) to give an overall assessment that the intervention is effective. Meta-

353 analyses tend not to combine different metrics because the resulting effect size would not be

354 linked to any metric and would therefore lose some meaning. Some studies that can be

355 considered by expert assessment also lack sufficient detail for the calculations involved in meta-

356 analysis (Haddaway and Verhoeven, 2015). Conservation Evidence highlights (e.g. in synopsis 
357 introductions) interventions or groups of interventions where we think conducting a meta-

358 analyses would be worthwhile as a more robust alternative to expert assessment.

\section{2.4. Accessing the database}

360 Outputs from each subject-wide evidence synthesis (interventions, summary paragraphs, key

361 messages, expert assessments) are freely available within the searchable online database,

362 www.conservationevidence.com. Users can search and filter the database in multiple ways,

363 including by taxon, habitat, intervention and threat. Synopses capture most of each subject-

364 wide evidence synthesis (interventions, summarized paragraphs and key messages) in a free-to-

365 download pdf, and in some cases as a printed book. An annual publication, What Works in

366 Conservation (e.g. Sutherland et al., 2018), presents the key messages and expert assessment

367 for interventions reviewed so far, as a rapid overview and gateway into the online database (via

368 hyperlinks).

\subsection{Updates}

370 The Conservation Evidence database is designed to allow the regular incorporation of new

371 evidence. Updating each subject-wide evidence synthesis involves searching new volumes,

372 issues, or documents within the originally-searched literature sources; searching additional

373 literature sources; and adding new interventions or adjusting existing ones (e.g. where new

374 literature suggests actions could be divided into multiple interventions). Further documents

375 suggested by users since the publication of the original synthesis can also be included. Thus, all

376 users can contribute studies to evidence syntheses through publishing their own articles and/or 
377 highlighting articles published by others. Key messages are updated and expert assessments 378 repeated for any interventions where new evidence was added. Conservation Evidence has 379 started to update existing syntheses and, in the short term, aims to produce updates every few

380 years. In the longer term, we envisage updating the database in near-real time as new evidence 381 is published, perhaps with the help of artificial intelligence to find publications and/or extract 382 data (Westgate et al. 2018).

\section{3. Discussion}

\section{3.1. Synthesizing complex evidence at scale}

385 The methods developed to build the Conservation Evidence database allow for the synthesis of 386 complex evidence across broad subjects and ultimately across whole disciplines. Using

387 discipline-wide searches and subject-wide syntheses, we can efficiently synthesize evidence for 388 both major and obscure topics, with a large or limited evidence base, respectively. Through a 389 combination of summary paragraphs, key messages and expert assessment, we can present a 390 general overview of the evidence incorporating a diversity of metrics, whilst allowing users to 391 drill down to the evidence most relevant to their situation. Key messages and expert 392 assessment can also highlight knowledge gaps and clusters for subjects and interventions. 393 Finally, by using short summary paragraphs in plain English, we produce a user-friendly end 394 product. We believe the truly unique feature of our methods is the combination of subject-wide 395 synthesis and discipline-wide searches: we are not aware of any other synthesis projects that 396 work across entire subjects and simultaneously collate literature for future syntheses within the 
397 discipline. We suggest these methods could be used to synthesize evidence for other themes

398 within biodiversity conservation, such as threats or monitoring methods.

The Conservation Evidence database complements other systematic evidence synthesis

400 outputs. The Conservation Evidence database provides syntheses of evidence over a broad

401 range of topics, for which the investment in a systematic review is not (yet) justified but

402 something more than just a map of the evidence would be useful. Systematic reviews, which

403 favor depth of review over breadth of topics reviewed, are desirable for interventions with a

404 large evidence base, where studies present contrasting results, for contentious topics, or where

405 the risks posed by an incorrect conclusion are severe (Collaboration for Environmental

406 Evidence, 2013; Collins et al., 2015). Systematic maps provide a rapid and inexpensive overview

407 of the state of evidence in a broad subject or topic, without detailing what the evidence finds.

408 They are most useful for identifying knowledge gaps and clusters, which can help direct

409 research effort where it is most needed (Haddaway et al. 2016). All of these systematic

410 outputs - including the Conservation Evidence database - are clearly organized, permanent,

411 searchable and designed to minimize several key sources of bias, especially compared to other

412 communication methods such as traditional literature reviews, notes from conference

413 presentations, or word of mouth.

\section{3.2. How the Conservation Evidence database helps to overcome barriers in} 415 research-implementation spaces

416 The Conservation Evidence database is designed to overcome some of the barriers between

417 conservation research, practice and policy (Table 1), facilitating the flow of information 
418 between (and within) these domains. For example, the scientific literature is vast and ever-

419 expanding, yet only a fraction of it is directly relevant to practitioners or policy-makers

420 (Westgate et al. 2018). The Conservation Evidence database helps to overcome this barrier by

421 presenting a relevant subset of the literature, containing quantitative information about the

422 effects of conservation interventions - which can be a key factor in making robust conservation

423 decisions (Adams and Sandbrook 2013; see also Section 3.4). Furthermore, the database is

424 categorized at multiple levels (subjects, interventions, individual studies), allowing users to

425 quickly drill down to relevant information, and combine it within and across levels to generate a

426 custom evidence synthesis. We are currently developing an online tool that allows users to

427 formally generate custom evidence syntheses.

$428 \quad$ A key feature of the Conservation Evidence database is its breadth, synthesizing

429 evidence for a large number of questions (interventions). Since the first Conservation Evidence

430 synthesis began in 2010, we have reviewed over 1,800 interventions (Sutherland et al., 2018)

431 for 15 subjects. The aim is to synthesize the evidence for the effects of all interventions, for all

432 taxa and all habitats, everywhere in the world.

433 Clearly there is a trade-off between breadth and depth of the database, so we cannot

434 claim to have captured all of the available evidence for each intervention. The assumption is

435 that users benefit from a synthesis of the evidence in the sources we search, as long as that is

436 based on an unbiased sample of the available evidence, and users understand that the evidence

437 base might be incomplete. A similar assumption implicitly, or explicitly, supports the use of

438 other forms of rapid evidence assessment (e.g. Collins et al., 2015). As we have already included 
439 studies from over 280 journals and grey literature sources in the database, we think we have

440 captured a substantial proportion of the relevant literature. We reduce publication and

441 geographic biases by searching grey and non-English literature.

442 We acknowledge that even if all the available journals and grey literature were screened

443 in multiple languages, a substantial amount of conservation-relevant knowledge would not be

444 captured and a knowledge-accessibility barrier remains. Many reports remain as internal

445 documents and are not publicly accessible. Moreover, some data on the effects of conservation

446 interventions are not formally reported and remains as case experience in minds and

447 notebooks. Although it may be possible to include case experience in the Conservation

448 Evidence database, for example through the use of interviews, it can be difficult to capture in a

449 systematic fashion and is likely subject to behavioral, social and cognitive biases that can be

450 difficult for third parties to assess. As such, we have decided not to include it in the database at

451 present. Still, such experiential and tacit knowledge should be used to complement the

452 Conservation Evidence database when making conservation decisions (Section 3.3).

\begin{tabular}{|l|l|l|}
\hline $\begin{array}{l}\text { Suggested barriers between } \\
\text { conservation research and } \\
\text { practice/policy }\end{array}$ & Example references & $\begin{array}{l}\text { How the Conservation } \\
\text { Evidence database helps to } \\
\text { overcome or lower this } \\
\text { barrier }\end{array}$ \\
\hline
\end{tabular}




\begin{tabular}{|c|c|c|}
\hline $\begin{array}{l}\text { Research produces } \\
\text { fragmented information that } \\
\text { often does not address } \\
\text { questions or problems } \\
\text { relevant to conservation } \\
\text { practice/policy. }\end{array}$ & $\begin{array}{l}\text { McNie, 2007; Roux et al., } \\
\text { 2006; Knight et al., } 2008 \text {; } \\
\text { Bainbridge, 2014; Gossa et } \\
\text { al., 2015; Rose et al., } 2018\end{array}$ & $\begin{array}{l}\text { Practitioners/policy-makers } \\
\text { suggest interventions to be } \\
\text { included in the database. } \\
\text { Interventions with little } \\
\text { evidence are highlighted for } \\
\text { researchers. Thus, the } \\
\text { database can act as a source } \\
\text { of inspiration for } \\
\text { practice/policy-relevant } \\
\text { research. The database } \\
\text { includes practice/policy- } \\
\text { relevant research e.g. } \\
\text { published in the journal } \\
\text { Conservation Evidence or } \\
\text { reports from conservation } \\
\text { organizations. }\end{array}$ \\
\hline $\begin{array}{l}\text { Practitioners/policy-makers } \\
\text { need answers more quickly } \\
\text { than they can be produced } \\
\text { by research, or even reviews } \\
\text { of existing research. }\end{array}$ & $\begin{array}{l}\text { Bainbridge, 2014; Gossa et } \\
\text { al., } 2015\end{array}$ & $\begin{array}{l}\text { Database is created } \\
\text { proactively, reviewing the } \\
\text { evidence for all } \\
\text { interventions before a } \\
\text { specific request from } \\
\text { practitioners/policy-makers. } \\
\text { Evidence synthesis } \\
\text { prioritizes breadth of } \\
\text { interventions covered over } \\
\text { depth of review for each } \\
\text { intervention to provide } \\
\text { some synthesized evidence } \\
\text { for all interventions, rather } \\
\text { than detailed synthesis for } \\
\text { few interventions. }\end{array}$ \\
\hline
\end{tabular}




\begin{tabular}{|c|c|c|}
\hline $\begin{array}{l}\text { Locating and accessing } \\
\text { relevant primary literature is } \\
\text { often too time-consuming } \\
\text { (due to the large volume of } \\
\text { published literature, } \\
\text { including much that is not } \\
\text { relevant to } \\
\text { practitioners/policy-makers) }\end{array}$ & $\begin{array}{l}\text { Pullin and Knight, 2005; } \\
\text { Gossa et al., 2015; } \\
\text { Westgate et al., } 2018\end{array}$ & $\begin{array}{l}\text { Database can be queried } \\
\text { using search terms or with } \\
\text { various filters (subjects, } \\
\text { countries, threats, actions). } \\
\text { Evidence within each subject } \\
\text { is organized in a consistent } \\
\text { way (interventions grouped } \\
\text { under threat and action } \\
\text { categories). }\end{array}$ \\
\hline $\begin{array}{l}\text { Even when primary } \\
\text { literature is located, reading } \\
\text { papers can be time } \\
\text { consuming, as much } \\
\text { research is not streamlined } \\
\text { for practitioners/policy- } \\
\text { makers. }\end{array}$ & $\begin{array}{l}\text { Pullin and Knight, 2005; } \\
\text { Bainbridge, 2014; } \\
\text { Westgate et al., } 2018\end{array}$ & $\begin{array}{l}\text { Database contains short, } \\
\text { summaries (usually <200 } \\
\text { words) of each study, plus } \\
\text { key messages to guide users } \\
\text { through the summary } \\
\text { paragraphs. }\end{array}$ \\
\hline $\begin{array}{l}\text { Much of the primary } \\
\text { literature is technical and } \\
\text { difficult to interpret for non- } \\
\text { specialists. Research is often } \\
\text { not communicated } \\
\text { effectively for non-scientists. }\end{array}$ & $\begin{array}{l}\text { Pullin and Knight, 2005; } \\
\text { Roux et al., 2006; } \\
\text { Bainbridge, 2014; Rose et } \\
\text { al., } 2018\end{array}$ & $\begin{array}{l}\text { Content of database is in } \\
\text { plain English, avoiding jargon } \\
\text { where possible (and } \\
\text { explaining it otherwise). }\end{array}$ \\
\hline $\begin{array}{l}\text { Primary literature may be in } \\
\text { a foreign language. }\end{array}$ & $\begin{array}{l}\text { Arlettaz et al., 2010; Gossa } \\
\text { et al., } 2015\end{array}$ & $\begin{array}{l}\text { Summaries are written in } \\
\text { English, even for primary } \\
\text { articles not in English. We } \\
\text { appreciate this introduces } \\
\text { language barriers for users } \\
\text { for whom English is a foreign } \\
\text { language. }\end{array}$ \\
\hline
\end{tabular}




\begin{tabular}{|c|c|c|}
\hline $\begin{array}{l}\text { Financial barriers can be } \\
\text { prohibitive (journal articles } \\
\text { are often hidden behind } \\
\text { paywalls, which can be too } \\
\text { expensive for conservation } \\
\text { practitioners/policy-makers; } \\
\text { books can also be too } \\
\text { expensive). }\end{array}$ & $\begin{array}{l}\text { Arlettaz et al., 2010; Gossa } \\
\text { et al., } 2015\end{array}$ & $\begin{array}{l}\text { Database and related } \\
\text { outputs are free to access. }\end{array}$ \\
\hline $\begin{array}{l}\text { Practitioners/policy-makers } \\
\text { do not trust that the } \\
\text { research, or synthesis, is } \\
\text { credible. }\end{array}$ & Bainbridge, 2014 & $\begin{array}{l}\text { Summary paragraphs include } \\
\text { key information (e.g. study } \\
\text { design, raw data, major } \\
\text { reported caveats) to allow } \\
\text { users to make some } \\
\text { judgement about study } \\
\text { quality (internal validity). } \\
\text { Methods used to produce } \\
\text { synthesis are reported } \\
\text { alongside the database. }\end{array}$ \\
\hline $\begin{array}{l}\text { The uptake of evidence is } \\
\text { often undermined by socio- } \\
\text { political agendas, whereby } \\
\text { practitioners/policy makers } \\
\text { tend to accept } \\
\text { information-or } \\
\text { disinformation - that } \\
\text { confirms pre-existing } \\
\text { worldviews but be critical of } \\
\text { evidence in conflict. }\end{array}$ & $\begin{array}{l}\text { Hameleers et al. 2019; } \\
\text { Ecker et al } 2019 .\end{array}$ & $\begin{array}{l}\text { A small contribution: the } \\
\text { Conservation Evidence } \\
\text { database serves as an } \\
\text { independent fact-checking } \\
\text { resource to help debunk } \\
\text { disproven or unfounded } \\
\text { claims. }\end{array}$ \\
\hline
\end{tabular}

455 The Conservation Evidence database aims to present scientific information in a format relevant 456 to practitioners and policy-makers who often struggle with the technical language, statistical 
457 analyses and length of scientific articles (Pullin and Knight 2005) - including systematic reviews

458 (Tricco et al. 2016). The database uses short paragraphs in plain English. The content is also

459 edited/reviewed by practitioners and policy-makers who sit on the synthesis advisory boards. In

460 addition, interventions are tagged (grouped) according to the IUCN universal classification

461 schemes of threats and actions (Salafsky et al., 2008), which were developed with input from

462 practitioners and therefore reflect their thought processes.

The database breaks down some language barriers by summarizing some articles

464 originally published in languages other than English, making them more accessible to English

465 speakers (and at least all in a common language). We take opportunities to translate syntheses

466 into alternative languages where possible (e.g. Hebrew for bee conservation), and have

467 incorporated Google Translate into our website for "on the fly" translation. We appreciate that

468 we still have work to do to break down language barriers for non-English-speaking users of the

469 Conservation Evidence database.

$470 \quad$ In many cases, the knowledge transfer barriers in the research-implementation space

471 arise upstream of evidence synthesis: there are often no (or few) scientific studies relevant to

472 practitioners or policy-makers. For example, scientists may focus on global analyses, complex

473 statistics and studies that push the boundaries of fundamental scientific knowledge to generate

474 publications with a high academic impact-but which are of little use to practitioners and

475 policy-makers (McNie, 2007; Hulme, 2011; Braunisch et al., 2012). To overcome this barrier,

476 practitioners and policy-makers contribute to shaping the interventions included in the

477 Conservation Evidence database. Furthermore, the database highlights knowledge gaps (i.e. 
478 which interventions are supported by no, little or low quality evidence) and clusters (e.g. in

479 certain locations or habitat types). Thus, researchers can see which questions are of interest to

480 practitioners and policy-makers, and which are lacking evidence-based answers. Being able to

481 demonstrate a knowledge gap for a practice- or policy-relevant question may help researchers

482 justify research funding.

483 There may also be psychological barriers limiting the flow of information between

484 research, practice and policy. For example, if institutionalized methods and relationships do not

485 currently involve interactions between research and practice, a certain degree of activation

486 energy will be needed to change habits (van Kerkhoff and Lebel, 2015). Further, scientific

487 evidence is often discounted when it challenges people's pre-existing values or worldviews,

488 especially when they are strongly connected to defined social identities (Roux et al., 2006;

489 Newell et al., 2014). Related to this, there is a growing availability of highly visible and

490 accessible, but often unreliable, information - especially on social media - which can "crowd

491 out" reliable sources of evidence (Ladle et al., 2005). Whilst many solutions to these problems

492 are largely outside of the scope of the Conservation Evidence project (e.g. detecting and

493 removing disinformation on social media platforms), we suggest that the Conservation

494 Evidence database may indirectly help to combat the spread of disinformation by increasing the

495 accessibility and visibility of verifiable research evidence (see also Section 3.4 on Evidence

496 Champions) and may help to reduce the impact of politically motivated disinformation by

497 providing an open, objective, independent fact-checking resource for practitioners (Ecker, et al., 498 2019; Hameleers and van der Meer, 2019). 
In an attempt to normalize use of the Conservation Evidence database, and reduce the

500 psychological barrier of using a new tool, the database is integrated into an increasing number

501 of practitioner-focused resources and decision-support software tools. It complements existing

502 information on the websites of the IUCN Red List (https://www.iucnredlist.org ), the National

503 Biodiversity Network (https://nbn.org.uk ), the British Trust for Ornithology

504 (https://www.bto.org), and the UNEP-Agreement on the Conservation of African-Eurasian

505 Migratory Waterbirds (https://www.unep-aewa.org/). The database is embedded in the

506 Conservation Management System software (https://www.software4conservation.com/cmsi-

507 software) used by Natural England and 10 other organizations to plan land management. The

508 Cool Farm Tool (https://coolfarmtool.org/) is used by major grocery retailers to help farmers

509 choose practices that reduce greenhouse gas emissions and, through the integration of the

510 Conservation Evidence database, could be beneficial for biodiversity.

Unfortunately, we currently have limited data about the effectiveness of Conservation

512 Evidence at breaking down barriers. Empirical evidence that the database can improve the

513 effectiveness of conservation when used is limited to one study. Walsh et al. (2014)

514 demonstrated that information synthesized by Conservation Evidence (on the effectiveness of

515 various interventions to control predators for bird conservation) changed practitioners' stated

516 choices of management in favor of more effective interventions, and away from interventions

517 that were likely to be ineffective or even harmful. Data on whether the Conservation Evidence

518 database increases the effectiveness of conservation in practice are difficult to collect, but we

519 are seeking research funding to do so (and encourage others to take up the challenge too). 
We do know that the website is well used: it received an average of 29,000 page views

521 per month between January and May 2019, by an average of 11,700 visitors per month from

522 over 220 countries and overseas territories. About $25 \%$ of visitors have used the website more

523 than once. Copies of What Works in Conservation have been read online or downloaded almost

52439,000 times as of June 2019.

$525 \quad$ Evidence Champions (see section 3.4) and others have provided feedback that the 526 database has helped their decision-making. For the AEWA (Agreement on the Conservation of 527 African-Eurasian Migratory Waterbirds) Secretariat, "it was a very helpful source, as we could

528 use it as a good reference and depending on the case also as a source for good examples on the 529 ground." The Rufford Foundation, who ask grant applicants to reference Conservation Evidence 530 in their applications, said, "I think that it has been valuable as a way of encouraging those 531 designing projects to look further afield to see if techniques they plan to use have been tried 532 before and, if yes, with what result. All of this has certainly helped our reviewers. Overall, 533 Conservation Evidence has... greatly improved the quality of the applications we receive." The Conservation Evidence database has been recognized in the political sphere. An 535 example from the Conservation Evidence database, publicized by Sutherland and Wordley 536 (2017), was used by Lord John Krebs to ask the UK government to ensure the government's 25 537 year environment plan would be evidence-based. The database has also been referred to in 538 multiple policy briefs and government documents, such as Defra's Consultation on the National 539 Pollinator Strategy (2014), The Scottish Government's Consultation on the Scotland Rural 
540 Development Programme (SRDP) 2014-2020, and The New Zealand Government's

541 Improvements to Biodiversity Assets Systems and Processes (2014).

543 3.3. Other tools in the Conservation Evidence toolbox that help overcome the 544 barriers in research-implementation spaces

The database is a core part of the wider Conservation Evidence project, which contains

547 other tools to help overcome barriers between conservation researchers, practitioners and

548 policy-makers. We briefly discuss these here.

The journal Conservation Evidence publishes research, monitoring results, and case

550 studies on the effects of conservation interventions. There is no requirement for novelty,

551 complex statistical analyses, or technical discussions. It is designed specifically to encourage

552 practitioners to submit their quantitative data and make them accessible to all. By converting

553 unpublished reports, internal documents and data from field notebooks into open access

554 publications, this journal helps overcome the knowledge-accessibility barrier discussed above

555 (Section 3.2). Providing an outlet for sharing robust, conservation-relevant primary research

556 could also encourage greater collaboration between researchers and practitioners.

558 organizations committed to using evidence (particularly the Conservation Evidence database)

559 when planning, funding, or publishing practical conservation actions, and/or testing a certain

560 number of interventions each year and publishing the results. These techniques are intended to

561 address some of the psychological barriers to the use of evidence (Section 3.2) by making a 
562 balanced assessment of evidence a routine and expected part of conservation planning.

563 Evidence Champions are supported through training in evidence interpretation and generation

564 techniques.

We also run more general workshops to explain what the Conservation Evidence

566 database is and how it can be used, or how practitioners can best carry out research to feed

567 into the database. Again, these can help to reduce behavioral or psychological barriers to the 568 use or production of conservation-relevant evidence.

570 3.4. Conservation in practice: other factors and actors in research571 implementation spaces

The Conservation Evidence database is built within the collaborative spaces occupied by

573 conservation researchers, practitioners and policy-makers. When the database is used to make

574 practical or policy conservation decisions, other actors (e.g. NGOs, governments, landowners,

575 farmers, indigenous communities, activists), issues (e.g. spiritual and cultural values, financial

576 resources, political), and information (e.g. the basic biology, distribution and status of species

577 and habitats, the presence and degree of threats, local knowledge and practical experience) are

578 introduced to these arenas (Roux et al., 2006; Toomey et al., 2017; Evans et al., 2018).

579 Conservation decisions are not made based on scientific evidence alone; socially acceptable

580 decisions must balance the needs of nature and people. In particular, the quantitative data

581 from the Conservation Evidence database will need to be combined with qualitative data, for

582 example derived from interviews or focus groups, to capture relevant tacit knowledge and 
583 values and ultimately design effective conservation strategies (Roux et al., 2006; Sutherland et 584 al. 2018).

Similarly, the Conservation Evidence database cannot tell practitioners or policy-makers

586 when or how to intervene. This decision will be influenced by site-specific issues and

587 information mentioned above, as well as assessments of the focal site's history and desired

588 future for all stakeholders. We recognize the potential that a list of interventions-some

589 assessed as beneficial to species or habitats - might encourage unnecessary active intervention.

590 In some cases, particularly in relatively intact sites, interventions may not be required to reach

591 a desired state and might do more harm than good to biodiversity. Thus, we caution against

592 assuming that intervening is always better than not intervening. To this end, we also include

593 some passive interventions in our syntheses (e.g. 'Allow shrubland to regenerate without active

594 management) to highlight that doing nothing is a management option to consider.

\subsection{Conclusion and Recommendations}

The Conservation Evidence database is assembled through a systematic, repeatable

597 process, with input from conservation researchers, practitioners and policy-makers. It is a

598 powerful and pragmatic tool to improve the use of scientific evidence by practitioners and

599 policy-makers, and encourage new research that is guided by practice and policy needs. The

600 database aims to complement existing evidence synthesis methods, and is complemented by

601 other tools within the Conservation Evidence toolbox, helping to create interactive spaces

602 where researchers, practitioners and other key stakeholders can collaboratively pursue

603 evidence-based conservation. 
Several concrete recommendations arise from our work building the Conservation

605 Evidence database and this article reflecting on the methods used to build it. Conservation

606 researchers, practitioners and policy-makers should consult the database when making

607 conservation decisions, to ensure those decisions are informed by evidence alongside expert

608 opinion, experience, local knowledge and values. Second, conservation intervention projects

609 should be monitored and the results published, whether or not successful and/or novel, in

610 order to strengthen the evidence base. Third, conservationists should engage with the

611 Conservation Evidence project, offer constructive feedback and help us to make the database

612 as useful as possible for you. Finally, the database should, and will, be constantly growing and

613 evolving as it incorporates new evidence, methodological improvements and technological

614 developments.

\section{References}

616 Adams, W., Sandbrook, C. 2013. Conservation, evidence and policy. Oryx, 47(3), 329-335.

617 doi:10.1017/S0030605312001470

618 Amano, T., González-Varo, J.P., Sutherland, W.J., 2016. Languages are still a major barrier to

619 global science. PLoS Biol. 14 (12), e2000933, doi:10.1371/journal.pbio.2000933.

620 Anon, 2007. The great divide. Nature, 450, 135-136, doi.org/10.1038/450135b 
623 Arlettaz, R., Schaub, M., Fournier, J., Reichlin, T.S., Sierro, A., Watson, J.E., Braunisch, V., 2010.

624 From publications to public actions: when conservation biologists bridge the gap between

625 research and implementation. BioSci. 60, 835-842, doi.org/10.1525/bio.2010.60.10.10.

626 Bainbridge, I., 2014. Practitioners Perspective: How can ecologists make conservation policy

627 more evidence based? Ideas and examples from a devolved perspective. J. Appl. Ecol. 51, 1153-

628 1158, doi:10.1111/1365-2664.12294.

629 Borah, R., Brown, A.W., Capers, P.L., Kaiser, K.A., 2017. Analysis of the time and workers

630 needed to conduct systematic reviews of medical interventions using data from the PROSPERO

631 registry. BMJ Open. 7, e012545, doi: 10.1136/bmjopen-2016-012545.

632 Braunisch, V., Home, R., Pellet, J., Arlettaz, R., 2012. Conservation science relevant to action: A

633 research agenda identified and prioritized by practitioners. Biol. Conserv. 153, 201-210,

634 doi:10.1016/j.biocon.2012.05.007

635 Cardinale, B. J., Duffy, J. E., Gonzalez, A., Hooper, D. U., Perrings, C., Venail, P., Narwani, A.,

636 Mace, G.M., Tilman, D., Wardle, D.A., Kinzig, A.P., Daily, G.C., Loreau, M., Grace, J.B.,

637 Larigauderie, A., Srivastava, D.S., Naeem, S., 2012. Biodiversity loss and its impact on humanity.

638 Nature 486, 59-67. doi.org/10.1038/nature11148

639 Cochrane Library, 2019. About Cochrane Clinical Answers. Available at

640 https://www.cochranelibrary.com/cca/about. Accessed 23 March 2019. 
641 Cohen, J. A., 1960. Coefficient of agreement for nominal scales. Educ. Psychol. Measure. 20, 37-

642 46, doi: $10.1177 / 001316446002000104$.

643 Collins, A.M., Coughlin, D., Miller, J., Kirk, S., 2015. The Production of Quick Scoping Reviews

644 and Rapid Evidence Assessments: A How to Guide. Available at

645 https://www.gov.uk/government/publications/the-production-of-quick-scoping-reviews-and-

646 rapid-evidence-assessments. Accessed March 2019.

647 Collaboration for Environmental Evidence, 2013. Guidelines for Systematic Review and

648 Evidence Synthesis in Environmental Management. Version 4.2. Environmental Evidence:

649 www.environmentalevidence.org/Documents/Guidelines/Guidelines4.2.pdf. Accessed March 6502019.

651 Dicks, L.V., Wright, H.L., Ashpole, J.E., Hutchison, J., McCormack, C.G., Livoreil, B., Zulka, K.P.,

652 Sutherland, W.J., 2016. What works in conservation? Using expert assessment of summarised

653 evidence to identify practices that enhance natural pest control in agriculture. Biodivers.

654 Conserv. 25, 1383-1399, doi: 10.1007/s10531-016-1133-7.

655 Dirzo, R., Young, H. S., Galetti, M., Ceballos, G., Isaac, N. J. B., Collen, B., 2014. Defaunation in

656 the Anthropocene. Sci. 345, 401-406, doi: 10.1126/science.1251817.

657 Dwan, K., Gamble, C., Williamson, P.R., Kirkham, J.J., 2013. Systematic review of the empirical

658 evidence of study publication bias and outcome reporting bias - an updated review. PloS ONE 659 8(7), e66844, doi:10.1371/journal.pone.0066844. 
660 Ecker, U., Ang, L.C. 2019. "Political Attitudes and the Processing of Misinformation Corrections," 661 Political Psychology, 40, 241-260. doi.org/10.1111/pops.12494

662 Evans, M.C., Davila, F., Toomey, A., Wyborn, C., 2017. Embrace complexity to improve 663 conservation decision making. Nat. Ecol. Evol. 1, 1588. doi.org/10.1038/s41559-017-0345-x

664 Farace, D.J., Schöpfel, J.S., 2010. Introduction Grey Literature. In, Farace, D.J., Schöpfel, J.S. 665 (eds.) Grey Literature in Library and Information Studies, De Gruyter Sauer Berlin/New York.

666 Gerber, L.R., 2016. Conservation triage or injurious neglect in endangered species recovery. 667 Proceed. Nat Acad. Sci. 113, 3563-3566, doi: 10.1073/pnas.1525085113.

668 Gossa, C., Fisher, M., Milner-Gulland, E.J., 2015. The research-implementation gap: how 669 practitioners and researchers from developing countries perceive the role of peer-reviewed 670 literature in conservation science. Oryx 49, 80-87, doi:10.1017/\$0030605313001634.

671 Haddaway, N.R., Woodcock, P., Macura, B., Collins, A., 2015. Making literature reviews more 672 reliable through application of lessons from systematic reviews. Conserv. Biol. 29, 1596-1605, 673 doi.org/10.1111/cobi.12541.

674 Haddaway NR, Bernes C, Jonsson BG, Hedlund K (2016) The benefits of systematic mapping to 675 evidence-based environmental management. Ambio, 45, 613-620. doi:10.1007/s13280-016676 0773-x. 
677 Haddaway, N.R., Kohl, C., Rebelo da Silva, N., Schiemann, J., Spök, A., Stewart, R., Sweet, J.B.,

678 Wilhelm, R., 2017. A framework for stakeholder engagement during systematic reviews and

679 maps in environmental management. Environ. Evid. 6, 11, doi 10.1186/s13750-017-0089-8.

680 Haddaway, N.R., Macura, B., 2018. The role of reporting standards in producing robust

681 literature reviews. Nat. Clim. Change 8, 444-453. doi.org/10.1038/s41558-018-0180-3

682 Haddaway, N.R., Verhoeven, J.T., 2015. Poor methodological detail precludes experimental

683 repeatability and hampers synthesis in ecology. Ecol. and Evol. 5, 4451-4454.

$684 \quad$ doi.org/10.1002/ece3.1722

685 Haddaway, N.R., Westgate, M.J., 2019. Predicting the time needed for environmental

686 systematic reviews and systematic maps. Conserv. Biol. 33, 434-443, doi:10.1111/cobi.13231.

687 Hameleers, M., van der Meer, T.G.L.A. 2019. Misinformation and Polarization in a High-Choice

688 Media Environment: How Effective Are Political Fact-Checkers? Comm. Res,

$689 \quad$ doi.org/10.1177/0093650218819671

690 Hulme, P.E., 2011. Practitioner's perspectives: introducing a different voice in applied ecology.

691 J. Appl. Ecol. 48, 1-2, doi:10.1111/j.1365-2664.2010.01938.x

692 Knight, A.T., Cowling, R.M., Rouget, M., Balmford, A., Lombard, A.T., Campbell, B.M., 2008.

693 Knowing but not doing: selecting priority conservation areas and the research-implementation

694 gap. Conserv. Biol. 22, 610-617, doi.org/10.1111/j.1523-1739.2008.00914.x. 
695 Ladle, R.J., Jepson, P., Whittaker, R.J., 2005. Scientists and the media: the struggle for legitimacy

696 in climate change and conservation science. Interdiscipl. Sci. Rev., 30(3), 231-240.

697 doi.org/10.1179/030801805X42036

698 McAuley, L., Tugwell, P., Moher, D., 2000. Does the inclusion of grey literature influence

699 estimates of intervention effectiveness reported in meta-analyses? Lancet 356, 1228-1231,

700 doi:10.1016/S0140-6736(00)02786-0.

701 McNie, E.C., 2007. Reconciling the supply of scientific information with user demands: An

702 analysis of the problem and review of the literature. Environ. Sci. Policy 10, 17-38,

703 doi.org/10.1016/j.envsci.2006.10.004.

704 Mukherjee, N., Huge, J., Sutherland, W.J., McNeill, J., van Opstal, M., Dahdouh-Guebas, F.,

705 Koedam, N., 2015. The Delphi technique in ecology and biological conservation: applications

706 and guidelines. Methods Ecol. Evol. 6, 1097-1109, doi:10.1111/2041-210X.12387.

707 Newell, B.R., McDonald R.I., Brewer, M., Hayes, B.K., 2014. The psychology of environmental

708 decisions. Annu. Rev. Env. Resour. 39, 443-467. doi.org/10.1146/annurev-environ-010713-

$709 \underline{094623}$

710 Pullin, A.S., Knight, T.M., 2005. Assessing conservation management's evidence base: a survey

711 of management-plan compilers in the United Kingdom and Australia. Conserv. Biol. 19, 1989-

712 1996, doi:10.1111/j.1523-1739.2005.00287.x. 
713 Pullin, A.S., Stewart, G.B., 2006. Guidelines for systematic review in conservation and

714 environmental management. Conserv. Biol. 20, 1647-1656, doi: 10.1111/j.1523-

715 1739.2006.00485.x.

716 Ripple, W.J., Wolf, C.W., Newsome, T.M., Galetti, M., Alamgir, M., Crist, E., Mahmoud, M.I.,

717 Laurance, W.F., 15,364 scientist signatories from 184 countries, 2017. World scientists' warning

718 to humanity: a second notice. BioSci. $67,1026-1028$, doi.org/10.1093/biosci/bix125.

719 Rose, D. C., Sutherland, W. J., Amano, T., González-Varo, J. P., Robertson, R. J., Simmons, B. I.,

720 Wauchope, H.S., Kovacs, E., Durán, A.P., Vadrot, A.B.M., Wu, W., Dias, M.P., Di Fonzo, M.M.I.,

721 Ivory, S., Norris, L., Nunes, M.H., Nyumba, T.O., Steiner, N., Vickery, J., Mukherjee, N., 2018. The

722 major barriers to evidence-informed conservation policy and possible solutions. Conserv. Lett.

723 11(5), e12564, doi.org/10.1111/conl.12564

724 Roux, D.J., Rogers, K.H., Biggs, H.C., Ashton, P.J., Sergeant, A., 2006. Bridging the science-

725 management divide: moving from unidirectional knowledge transfer to knowledge interfacing

726 and sharing. Ecol. Soc. 11, 4. [online] URL: http://www.ecologyandsociety.org/vol11/iss1/art4/

727 Salafsky, N., Salzer, D., Stattersfield, A.J., Hilton-Taylor, C., Neugarten, R., Butchart, S.H., Collen,

728 B., Cox, N., Master, L.L., O'Connor, S., Wilkie, D., 2008. A standard lexicon for biodiversity

729 conservation: unified classifications of threats and actions. Conserv. Biol. 22, 897-911,

730 doi:10.1111/j.1523-1739.2008.00937.x.

731 Smith, R.K., Sutherland, W.J., 2014. Amphibian Conservation: Global Evidence for the Effects of

732 Interventions. Pelagic Publishing, Exeter, UK. 
733 Soulé, M.E., 1985. What is conservation biology? BioSci. 35, 727-734, doi: 10.2307/1310054.

734 Stewart G, Ward J. 2019. Meta-science urgently needed across the environmental nexus: a

735 comment on Berger-Tal et al. Behav. Ecol. 30(1) 9-10. doi.org/10.1093/beheco/ary155

736 Sutherland, W.J., Dicks, L.V., Everard, M., Geneletti, D., 2018. Qualitative methods for ecologists

737 and conservation scientists. Methods Ecol. Evol., 9(1), 7-9. DOI: 10.1111/2041-210X.12956

738 Sutherland, W.J., Dicks, L.V., Ockendon, N., Petrovan, S.O., Smith, R.K., 2018. What Works in

739 Conservation 2018. Open Book Publishers, Cambridge, UK. doi.org/10.11647/OBP.0131.13

740 Sutherland, W.J., Pullin, A.S., Dolman, P.M., Knight, T.M., 2004. The need for evidence-based

741 conservation. TREE. 19, 305-308, doi:10.1016/j.tree.2004.03.018.

742 Sutherland, W.J., Wordley, C.F.R., 2017. Evidence complacency hampers conservation. Nature

743 Ecol. Evol., 1, 1215-1216. doi.org/10.1038/s41559-017-0244-1

744 Sutherland, W.J., Wordley, C.F.R., 2018. A fresh approach to evidence synthesis. Nature 558,

745 364-366, doi:10.1038/d41586-018-05472-8.

746 Toomey, A.H., Knight, A.T., Barlow, J., 2017. Navigating the space between research and

747 implementation in conservation. Conserv. Lett. 10, 619-625, doi:10.1111/conl.12315.

748 Tricco, A.C., Cardoso, R., Thomas, S.M., Motiwala, S., Sullivan, S., Kealey, M.R., Hemmelgarn, B.,

749 Ouimet, M., Hillmer, M.P., Perrier, L., Shepperd, S., Straus S.E. (2016) Barriers and facilitators to 
750 uptake of systematic reviews by policy makers and health care managers: a scoping review.

751 Implementation Science, 11:4, doi: 10.1186/s13012-016-0370-1.

752 van Kerkhoff, L.E., Lebel, L., 2015. Coproductive capacities: rethinking science-governance

753 relations in a diverse world. Ecol. Soc. 20(1): 14, doi:10.5751/ES-07188-200114

754 Walsh, J.C., Dicks, L.V., Sutherland, W.J., 2014. The effect of scientific evidence on conservation

755 practitioners' management decisions. Conserv. Biol. 29, 88-98, doi.org/10.1111/cobi.12370.

756 Westgate, M. J., Haddaway, N. R., Cheng, S. H., Mclntosh, E. J., Marshall, C., Lindenmayer, D. B.,

757 2018. Software support for environmental evidence synthesis. Nat. Ecol. Evol. 2, 588-590.

758 doi.org/10.1038/s41559-018-0502-x

759 Williams, D.R., Pople, R.G., Showler, D.A., Dicks, L.V., Child, M., zu Ermgassen, E.K.H.J.,

760 Sutherland, W.J., 2013. Bird Conservation: Global Evidence for the Effects of Interventions.

761 Pelagic Publishing, Exeter, UK.

762 Young, K.D., Van Aarde, R.J., 2011. Science and elephant management decisions in South Africa.

763 Biol. Conserv. 144, 876-885, doi.org/10.1016/j.biocon.2010.11.023. 
772 Figure 1. An overview of the methods used to build the Conservation Evidence database

773 (discipline-wide literature searches and subject-wide evidence synthesis), and how a range of

774 end users are incorporated into the construction process. Rectangles represent processes, and

775 rhomboids represent outputs. Numbers indicate section of text where item is explained.

776

777

778 Table 1: Some barriers that inhibit interaction between research and practice/policy, and how 779 the Conservation Evidence database helps to overcome or lower these barriers. 
Appendix 


\section{General format for Conservation Evidence summary paragraphs:}

A [TYPE OF STUDY ${ }^{1}$ ] in [YEARS X-Y] in [HOW MANY SITES] in/of [HABITAT] in [REGION and COUNTRY] [REFERENCE] found that [INTERVENTION] [SUMMARY OF ALL KEY RESULTS ${ }^{2}$ ] for [SPECIES/HABITAT TYPE]. [DETAILS OF KEY RESULTS, INCLUDING DATA ${ }^{2}$ ]. In addition, [EXTRA RESULTS, IMPLEMENTATION OPTIONS, CONFLICTING RESULTS]. The [DETAILS OF EXPERIMENTAL DESIGN, INTERVENTION METHODS and KEY DETAILS OF SITE CONTEXT ${ }^{3}$ ]. Data was collected in [DETAILS OF SAMPLING METHODS].

1. Type of study is described using standard terms, available alongside the Conservation Evidence database and in synopses.

2. Results (e.g. difference between groups, changes over time) are statistically significant, unless specifically stated (e.g. with the sentence "These results were not tested for statistical significance").

3. For the sake of brevity, only nuances essential to the interpretation of the results can be included. The reader is always encouraged to read the original source to get a full understanding of the study site (e.g. history of management, physical conditions).

\section{For example:}

A replicated, controlled study in 2001 of bottomland hardwood forest in Georgia, USA (1) found that prescribed burning had no significant effect on abundance, diversity or richness of amphibians. Abundance did not differ significantly at burned and unburned sites for all amphibians (burned: 43; unburned: 62), salamanders (2 vs 6) or frogs and toads (39 vs 50). The same was true for species richness overall (burned: 8; unburned: 8 species), for salamanders ( 2 vs 2 ) and frogs and toads ( 6 vs 6 ). The volume of coarse woody debris was similar in burned $\left(60 \mathrm{~m}^{3} / \mathrm{ha}\right)$ and unburned stands $\left(128 \mathrm{~m}^{3} / \mathrm{ha}\right)$. Amphibians were monitored in three winter-burned and unburned stands from July to October 2001. Drift-fencing with pitfall traps, artificial cover boards and PVC pipe refugia were randomly placed within each site.

(1) Moseley K.R., Castleberry S.B. \& Schweitzer S.H. (2003) Effects of prescribed fire on herpetofauna in bottomland hardwood forests. Southeastern Naturalist, 2, 475-486.

Figure A1. The general format for Conservation Evidence summary paragraphs with an example. 
Table A1. Overall effectiveness categories, with colors as used in Sutherland et al. (2018). Reproduced with permission from Sutherland et al. (2018).

\begin{tabular}{|c|c|c|c|}
\hline Category & Description & General criteria & Thresholds \\
\hline Beneficial & $\begin{array}{l}\text { Effectiveness has been } \\
\text { demonstrated by clear } \\
\text { evidence. Expectation of } \\
\text { harm is small compared with } \\
\text { the benefits }\end{array}$ & $\begin{array}{l}\text { High median benefit score } \\
\text { High median certainty } \\
\text { score } \\
\text { Low median harm score }\end{array}$ & $\begin{array}{l}\text { Effectiveness: }>60 \% \\
\text { Certainty: }>60 \% \\
\text { Harm: }<20 \%\end{array}$ \\
\hline $\begin{array}{l}\text { Likely to be } \\
\text { beneficial }\end{array}$ & $\begin{array}{l}\text { Effectiveness is less well } \\
\text { established than for those } \\
\text { listed under 'effective' } \\
\text { OR } \\
\text { There is clear evidence of } \\
\text { medium effectiveness }\end{array}$ & $\begin{array}{l}\text { High benefit score } \\
\text { Lower certainty score } \\
\text { Low harm score } \\
\text { OR } \\
\text { Medium benefit score } \\
\text { High certainty score } \\
\text { Low harm score }\end{array}$ & $\begin{array}{l}\text { Effectiveness: }>60 \% \\
\text { Certainty: } 40-60 \% \\
\text { Harm: }<20 \% \\
\text { OR } \\
\text { Effectiveness: } 40-60 \% \\
\text { Certainty: } \geq 40 \% \\
\text { Harm: }<20 \%\end{array}$ \\
\hline $\begin{array}{l}\text { Trade-off between } \\
\text { benefit and harms }\end{array}$ & $\begin{array}{l}\text { Interventions for which } \\
\text { practitioners must weigh up } \\
\text { the beneficial and harmful } \\
\text { effects according to } \\
\text { individual circumstances and } \\
\text { priorities }\end{array}$ & $\begin{array}{l}\text { Medium benefit and } \\
\text { medium harm scores } \\
\text { OR } \\
\text { High benefit and high } \\
\text { harm scores } \\
\text { High certainty score }\end{array}$ & $\begin{array}{l}\text { Effectiveness: } \geq 40 \% \\
\text { Certainty: } \geq 40 \% \\
\text { Harm: } \geq 20 \%\end{array}$ \\
\hline $\begin{array}{l}\text { Unknown } \\
\text { effectiveness } \\
\text { (limited evidence) }\end{array}$ & $\begin{array}{l}\text { Currently insufficient data, } \\
\text { or data of inadequate quality }\end{array}$ & Low certainty score & $\begin{array}{l}\text { Effectiveness: Any } \\
\text { Certainty: }<40 \% \\
\text { Harm: Any }\end{array}$ \\
\hline $\begin{array}{l}\text { Unlikely to be } \\
\text { beneficial }\end{array}$ & $\begin{array}{l}\text { Lack of effectiveness is less } \\
\text { well established than for } \\
\text { those listed under 'likely to } \\
\text { be ineffective or harmful' }\end{array}$ & $\begin{array}{l}\text { Low benefit score } \\
\text { Medium certainty score } \\
\text { and/or some variation } \\
\text { between experts }\end{array}$ & $\begin{array}{l}\text { Effectiveness: }<40 \% \\
\text { Certainty: } 40-60 \% \\
\text { Harm: }<20 \%\end{array}$ \\
\hline $\begin{array}{l}\text { Likely to be } \\
\text { ineffective or } \\
\text { harmful }\end{array}$ & $\begin{array}{l}\text { Ineffectiveness or } \\
\text { harmfulness has been } \\
\text { demonstrated by clear } \\
\text { evidence }\end{array}$ & $\begin{array}{l}\text { Low benefit score } \\
\text { High certainty score } \\
\text { (regardless of harm) } \\
\text { OR } \\
\text { Low benefit score } \\
\text { High harm score } \\
\text { (regardless of certainty of } \\
\text { effectiveness) }\end{array}$ & $\begin{array}{l}\text { Effectiveness: }<40 \% \\
\text { Certainty: }>60 \% \\
\text { Harm: Any } \\
\text { OR } \\
\text { Effectiveness: }<40 \% \\
\text { Certainty: } \geq 40 \% \\
\text { Harm: } \geq 20 \%\end{array}$ \\
\hline
\end{tabular}




\section{Glossary}

Definitions of terms as used by Conservation Evidence and in the main article text.

Discipline: A research field of a size that could be taught as a standalone undergraduate degree course, such as biodiversity conservation. Contains multiple subject areas.

Evidence synthesis: The process of combining multiple sources of evidence addressing a particular area or question (verb) or any product arising from this process (noun).

Expert assessment: Using multiple experts to assess the effectiveness, associated certainty and harm of an intervention, to produce a generalized overall effectiveness category for the intervention.

Intervention: A conservation action or management option that is currently used, or could be used, with the intention of benefitting biodiversity. For example, 'Use streamer lines to reduce seabird bycatch on longlines' or 'Legally protect habitat'.

Key messages: In the Conservation Evidence database, these provide an overview of the studies that tested a particular intervention. Intended to guide users to the more detailed study summaries.

Practitioner: Decision-maker whose main occupation is not research, but the implementation of actions in the field, for example conservation actions to protect and manage natural resources (Gossa et al., 2015).

Policy-maker: Decision-maker whose main occupation is not research, but defining plans and legislation, for example to protect and sustainably manage natural resources.

Research-implementation gap: A conceptual division or void between research and practice or policy, whereby interaction or flow of information between these groups is limited. Encourages a focus on linear transfer of absolute truth from science to practice and policy, rather than recognizing the complex and multi-directional interactions between these groups (Toomey et al., 2017). 
Research-implementation space: A conceptual arena in which researchers, practitioners and/or policy-makers interact and work. Barriers within this space can hinder interactions and knowledge transfer. Research-implementation spaces may also overlap with factors such as local knowledge, beliefs and societal values, and these factors should be considered when making conservation decisions.

Study summary: A paragraph summarizing the conceptually distinct part (or the whole) of a paper, report or other source of evidence. Written using a structured methodology to present the main methods and results.

Subject: A large area within a discipline, big enough to comprise multiple (usually hundreds) of related review questions. Subjects within the Conservation Evidence project include bird conservation, conservation of forest vegetation, and management of aquatic invasive species.

Subject-wide evidence synthesis: A systematic method of evidence synthesis that reviews closed review questions across entire subject areas at once (verb) or any product arising from this process (noun). Necessitates trading-off depth of each review for breadth of topics covered. Will usually focus on one type of review question within the subject e.g. effects of interventions, impacts of threats, comparison of methods.

Synopsis: A document capturing the synthesized evidence for an entire subject (e.g. bird conservation). Conservation Evidence synopses contain the list of interventions, study summaries, key messages and background information and are published as pdfs and/or books.

\section{References}

Gossa, C., Fisher, M., Milner-Gulland, E.J., 2015. The research-implementation gap: how practitioners and researchers from developing countries perceive the role of peer-reviewed literature in conservation science. Oryx 49, 80-87, doi: 10.1017/S0030605313001634.

Toomey, A.H., Knight, A.T., Barlow, J., 2017. Navigating the space between research and implementation in conservation. Conserv. Lett. 10, 619-625, doi: 10.1111/conl.12315. 